\title{
Simultaneous medullary carcinoma, papillary carcinoma and granulomatous inflammation of the thyroid
}

Kamal Kataria ${ }^{1}$, MS, Rajni $\underline{\text { Yadav }}^{2}$, MD, Chitra $\underline{\text { Sarkar }}^{2}$, MD, Asis Kumar $\underline{K a r a k}^{2}$, MD

\begin{abstract}
Thyroid tumours with both papillary and medullary carcinoma features are rare and represent less than $1 \%$ of all thyroid malignancies. These tumours have a different clinical presentation and biological behaviour from tumours that have only papillary or medullary carcinoma features. The phenomenon of mixed thyroid tumours can be observed in two settings - a mixed tumour showing dual differentiation, or a collision tumour. For a precise diagnosis of this rare mixed thyroid carcinoma, fine needle aspiration cytology results should be correlated with serum calcitonin and thyroglobulin levels. The diagnosis should also be confirmed using immunocytochemistry. Surgery is the treatment of choice, and the role of postoperative radioiodine is controversial. We herein report the case of a 35-year-old man with a mixed medullary-papillary carcinoma of the thyroid, which presented with C-cell hyperplasia, granulomatous inflammation and metastasis to the cervical lymph nodes. The patient was treated with total thyroidectomy and nodal clearance. This case highlights the need for awareness of coexistent entities as they warrant separate treatments.
\end{abstract}

Keywords: calcitonin, granulomatous, medullary carcinoma, papillary carcinoma, thyrog/obulin

\section{INTRODUCTION}

Thyroid carcinomas are divided into two main groups based on their embryonic origin: parafollicular or medullary carcinomas (i.e. those that arise from the neuroectodermal derivation of the fourth branchial arch), and follicular and papillary carcinomas (i.e. those of foregut endodermal origin that arise from the base of the tongue). Papillary thyroid carcinoma (PTC) is the most common (75\%-80\%) histological type of thyroid cancer, ${ }^{(1)}$ whereas medullary thyroid carcinoma (MTC) represents only $5 \%-8 \%$ of cases. ${ }^{(2)}$ Tumours showing both features are rare (less than $1 \%$ of all thyroid malignancies), ${ }^{(3)}$ and have different clinical presentation and biological behaviour. This rare phenomenon may be observed in two settings - a mixed tumour showing dual differentiation $^{(4)}$ or a collision tumour (i.e. a tumour with two separate and different components). ${ }^{(5)}$ To date, less than 40 cases of mixed medullary-papillary carcinoma have been reported in the literature. ${ }^{(6)}$

We report a case of a collision tumour occurring in the right thyroid lobe. The collision tumour had lesions with features of MTC and PTC at two different locations that were separated by normal thyroid tissue. In addition, a separate focus of papillary carcinoma in the left thyroid lobe, C-cell hyperplasia with granulomatous inflammation in the right thyroid lobe and metastasis of the medullary component to the right neck lymph nodes were also seen. To the best of our knowledge, this is the first report of such a combination of features.

\section{CASE REPORT}

A 35-year-old man presented with complaints of a slowgrowing left thyroid swelling that had persisted for six months. He had no symptoms of hypo- or hyperthyroidism, dysphagia, voice change, abdominal pain, irradiation in the head and neck region, or a family history of thyroid cancer. He had a history of pulmonary tuberculosis one year before presentation.

Examination revealed a small, firm-to-hard, non-tender nodule measuring $2 \mathrm{~cm} \times 1.5 \mathrm{~cm}$ in the left lobe of the thyroid, and an enlarged level II cervical lymph node measuring $2.5 \mathrm{~cm}$ on right side of the neck. Ultrasonography of the patient's neck suggested the presence of an enlarged heterogenous mass, measuring $1.5 \mathrm{~cm} \times 1.0 \mathrm{~cm} \times 0.8 \mathrm{~cm}$, in the left lobe of the thyroid and a heterogeneous hypoechoic nodule, measuring $1.0 \mathrm{~cm} \times 0.8 \mathrm{~cm} \times 0.5 \mathrm{~cm}$, in the right lobe of the thyroid. Enlarged level II and level IV lymph nodes were also observed on the right side of the patient's neck. Fine needle aspiration cytology (FNAC) of the thyroid nodules and right neck lymph nodes were performed. Microscopy of the lymph node aspirate showed singly scattered cells with round to oval nuclei that had 'salt and pepper' chromatin (Fig. 1a), whereas aspiration of the thyroid nodule yielded only blood. A possible diagnosis of neuroendocrine tumour was made. Other than a mildly elevated serum calcitonin level, all haematological and serological investigations were within normal limits.

${ }^{1}$ Department of Surgical Disciplines, ${ }^{2}$ Department of Pathology, All India Institute of Medical Sciences, New Delhi, India.

Correspondence: Dr Rajni Yadav, Senior Resident, Room No. 334, Resident Doctors Hostel, Masjid Moth, All India Institute of Medical Sciences, New Delhi 110029, India.drrajniyadav@gmail.com 

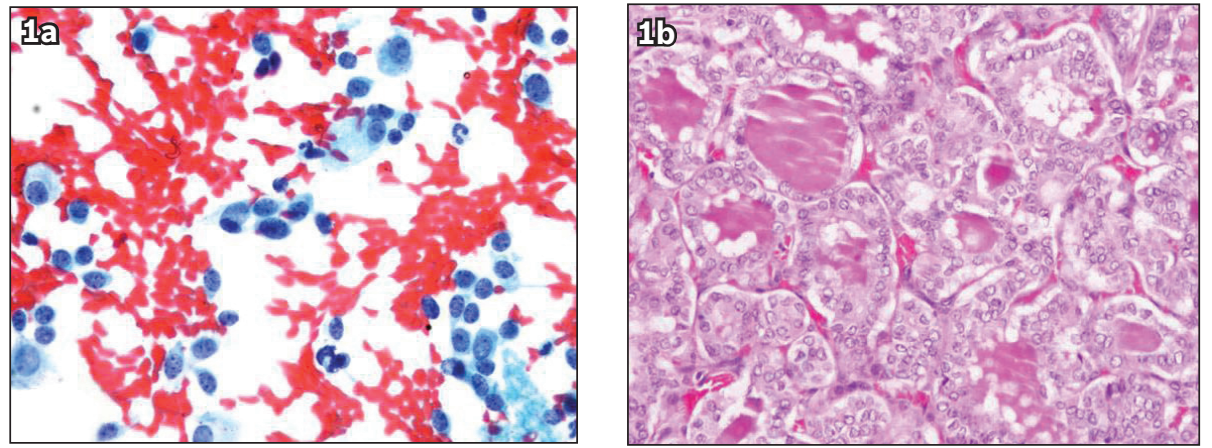

Fig. 1 Photomicrographs show (a) fine needle aspiration cytology smear of the right cervical lymph node, which shows singly scattered cells with 'salt and pepper' chromatin (Pap, $\times 400$ ); and (b) focus of papillary carcinoma in the left lobe of the thyroid, which shows follicular arrangement of tumour cells with orphan annie nuclei (Haematoxylin \& eosin, × 200).
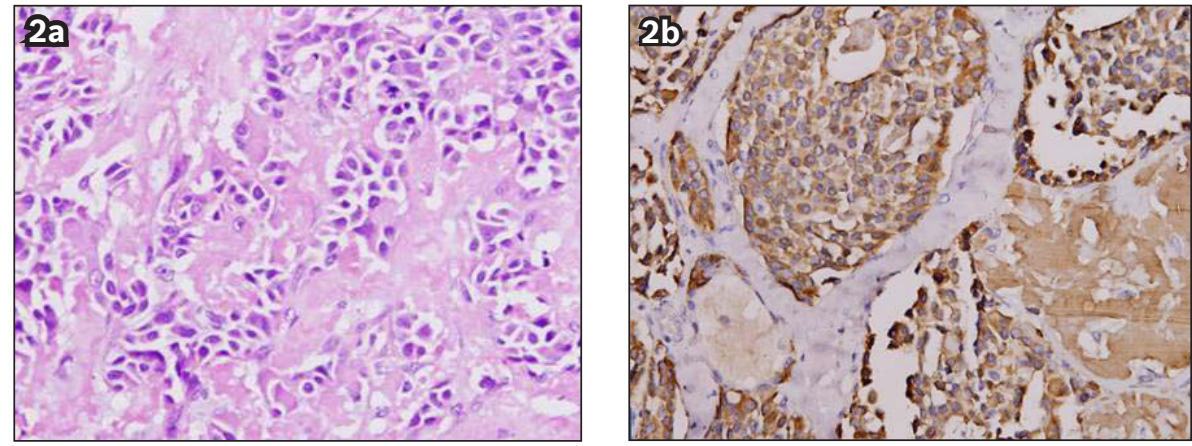

Fig. 2 Photomicrographs show that (a) the medullary carcinoma in right lobe of thyroid is composed of nests of tumour cells with stippled chromatin and interspersed amyloid (Hematoxylin and eosin, x 200); and (b) the medullary carcinoma component stains positive for calcitonin (× 200).

The patient underwent total thyroidectomy with modified right and central cervical nodal clearance. Grossly, the right and left thyroid lobes each had a solid greyish-white nodule measuring $1 \mathrm{~cm}$ and $1.5 \mathrm{~cm}$, respectively. Microscopic examination of the paraffin-embedded sections of the left thyroid nodule showed that it was a follicular variant of papillary carcinoma (Fig. 1b). Another focus of papillary carcinoma, also a follicular variant, was seen microscopically in the right thyroid lobe; this focus was not identified during gross examination. The medullary carcinoma (Fig. 2a), seen in the nodule of the right thyroid lobe, was immunopositive for calcitonin (Fig. 2b), chromogranin, synaptophysin and carcinoembryonic antigen, and immunonegative for thyroglobulin. C-cell hyperplasia (Fig. 3) and amyloid deposition were also seen in conjunction with the medullary carcinoma in the right thyroid lobe. The foci of papillary and medullary carcinoma in the right thyroid lobe were separated by normal thyroid parenchyma. In addition, necrotising granulomatous inflammation (Fig. 4) was also observed in the right thyroid lobe. Stains for tubercle bacillus and fungus were negative. Examination of the right cervical nodes revealed metastasis of MTC.

The postoperative period was uneventful, and follow-up revealed no rise in the patient's serum thyroglobulin and calcitonin levels. The patient did not have other stigmata of multiple endocrine neoplasia 2 (MEN2) syndrome. As polymerase chain reaction for tuberculosis was positive on evaluation, and since the most common cause of necrotising granulomatous inflammation in India is tuberculosis, the patient was started on antitubercular therapy. The patient refused genetic testing due to financial constraints. At follow-up a year post surgery, no locoregional recurrence was observed.

\section{DISCUSSION}

Mixed medullary-papillary carcinomas are very rare. Most reports of thyroid carcinomas are of either the medullary or papillary type. Mixed medullary-papillary carcinomas were first described in the early $1980 s^{(6)}$ Lax et al ${ }^{(7)}$ reported three cases in 1994, while Papotti et al ${ }^{(8)}$ described an important series of 11 cases of mixed medullary-follicular cell thyroid carcinomas in 1997. In MTCs, which account for about $5 \%-8 \%$ of all thyroid carcinomas, ${ }^{(2)} 3 \%-5 \%$ of the cases may display mixed features, with either a follicular or papillary component in the primary tumour, metastases, or both. ${ }^{\left({ }^{8}\right)}$ This association remains extremely rare and represents less than $0.5 \%$ of all thyroid tumours. ${ }^{(8)}$ Our case highlights the unique and rare coexistence of papillary carcinoma in both lobes of the thyroid, with medullary carcinoma and granulomatous inflammation present in the right thyroid lobe.

Various hypotheses explaining the origin of mixed medullary-follicular cell thyroid carcinoma have been documented. Some authors believe that neoplastic transformation of uncommitted stem cells leads to the simultaneous development of medullary and follicular neoplasms. This hypothesis is supported by the coexpression 


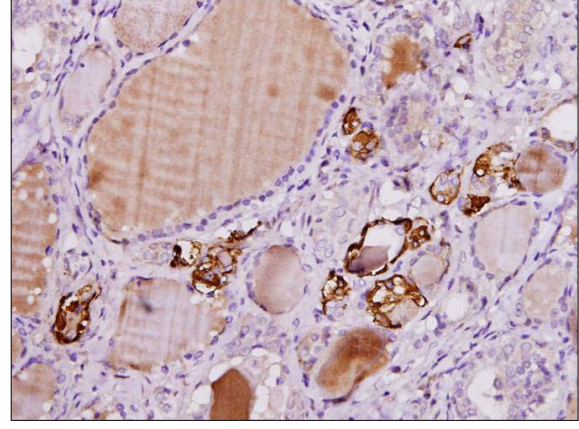

Fig. 3 Photomicrograph shows C-cell hyperplasia, highlighted by positive immunostaining for calcitonin ( $\times 200)$

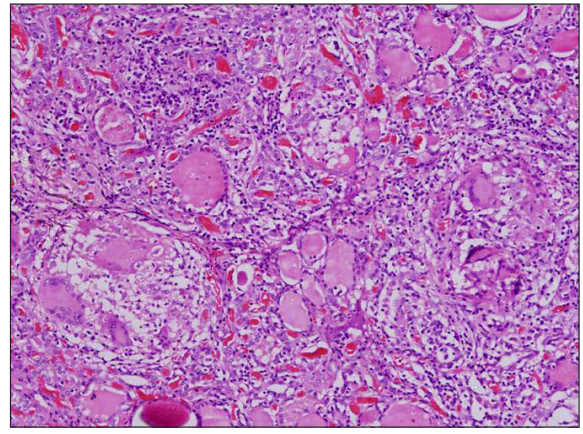

Fig. 4 Photomicrograph shows granulomas with giant cells (Hematoxylin and eosin, $\times 100)$.

of thyroglobulin and calcitonin in the tumour cells. ${ }^{(9,10)}$ Another possible hypothesis is that a common oncogenic stimulus leads to the simultaneous transformation of both follicular and C-cell progenitor stem cells, and thus the formation of both medullary and follicular compounds. ${ }^{(11)}$ Burstein et $\mathrm{al}^{(12)}$ and Preto et $\mathrm{al}^{(13)}$ suggested that the multipotentiality of main cells in a solid nest may be responsible for the histogenesis of C-cells, follicular cells, and also, thyroid tumours. Volante et al, ${ }^{(14)}$ however, proposed the 'hostage hypothesis', which states that the neoplastic transformation of C-cells leads to the development of an MTC with entrapment of normal follicles among the cancerous cells. They proposed that the microenvironment provided by the MTC cells would subsequently stimulate the proliferation of the trapped follicular cells, resulting in hyperplastic and adenomatous follicles, which would ultimately acquire a fully developed neoplastic phenotype (either follicular or papillary) capable of producing lymph node or distant metastases. ${ }^{(14)}$

Granulomatous inflammation of the thyroid can be seen in de Quervain's thyroiditis, palpation thyroiditis, tuberculosis, sarcoidosis, mycosis and after thyroid surgery. A clinical and investigative workup helps to ascertain its exact aetiology. Diagnosis of a mixed medullary-papillary thyroid carcinoma by FNAC is extremely difficult, and sampling error can lead to a misdiagnosis. Therefore, FNAC results should be correlated with serum calcitonin and thyroglobulin levels, and diagnosis should be confirmed using immunocytochemistry. Treatment of mixed medullary-papillary carcinoma depends on the medullary component of the carcinoma. Surgical resection of the thyroid tumour and areas of its lymphatic drainage (levels II to VII), with preservation of the laryngeal nerve, is the treatment of choice. The role of adjuvant radioiodine in the treatment of mixed tumours is controversial because while the medullary component does not respond to radioiodine, the thyroglobulin immunoreactive component responds with regression, suggesting that adjuvant therapy may be efficacious. ${ }^{(15)}$

A precise diagnosis of this uncommon variety of mixed thyroid carcinoma is crucial for both adequate patient treatment and genetic screening to exclude MEN2 syndrome and familial MTC.

\section{REFERENCES}

1. Rosai J. Handling of thyroid follicular patterned lesions. Endocr Pathol 2005; 16:279-83.

2. Ball DW. Medullary thyroid cancer: therapeutic targets and molecular markers. Curr Opin Oncol 2007; 19:18-23.

3. Rossi S, Fugazzola L, De Pasquale L, et al. Medullary and papillary carcinoma of the thyroid gland occurring as a collision tumour: report of three cases with molecular analysis and review of the literature. Endocr Relat Cancer 2005; 12:281-9.

4. Marsh DJ, Learoyd DL, Andrew SD, et al. Somatic mutations in the RET proto-oncogene in sporadic medullary thyroid carcinoma. Clin Endocrinol (Oxf) 1996; 44:249-57.

5. Gero MJ, Lipper S, Chernys AE, Silver L. Medullary and papillary carcinomas occurring as a collision tumour: report of a case. Clin Nucl Med 1989; 14:171-4.

6. Nangue C, Bron L, Portmann L, et al. Mixed medullary-papillary carcinoma of the thyroid: report of a case and review of the literature. Head Neck 2009; 31:968-74.

7. Lax SF, Beham A, Kronberger-Schönecker D, Langsteger W, Denk $\mathrm{H}$. Coexistence of papillary and medullary carcinoma of the thyroid gland-mixed or collision tumour? Clinicopathological analysis of three cases. Virchows Arch 1994; 424:441-7.

8. Papotti M, Negro F, Carney JA, Bussolati G, Lloyd RV. Mixed medullary-follicular carcinoma of the thyroid. A morphological, immunohistochemical and in situ hybridization analysis of 11 cases. Virchows Arch 1997; 430:397-405.

9. Noel M, Delehaye MC, Segond N, et al. Study of calcitonin and thyroglobulin gene expression in human mixed follicular and medullary thyroid carcinoma. Thyroid 1991; 1:249-56.

10. Holm R, Sobrinho-Simões M, Nesland JM, Sambade C, Johannessen JV. Medullary thyroid carcinoma with thyroglobulin immunoreactivity. A special entity? Lab Invest 1987; 57:258-68.

11. Zhang P, Zuo H, Ozaki T, Nakagomi N, Kakudo K. Cancer stem cell hypothesis in thyroid cancer. Pathol Int 2006; 56:485-9.

12. Burstein DE, Nagi C, Wang BY, Unger P. Immunohistochemical detection of p53 homolog p63 in solid cell nests, papillary thyroid carcinoma, and Hashimoto's thyroiditis: a stem cell hypothesis of papillary carcinoma oncogenesis. Hum Pathol 2004; 35:465-73.

13. Preto A, Cameselle-Teijeiro J, Moldes-Boullosa J, et al. Telomerase expression and proliferative activity suggest a stem cell role for thyroid solid cell nests. Mod Pathol 2004; 17:819-26.

14. Volante M, Papotti M, Roth J, et al. Mixed medullary follicular thyroid carcinoma: molecular evidence for a dual origin of tumour components. Am J Pathol 1999; 55:1499-509.

15. Papotti $M$, Volante $M$, Komminoth $P$, Sobrinho-Simões $M$, Bussolati G. Thyroid carcinomas with mixed follicular and C-cell differentiation patterns. Semin Diagn Pathol 2000; 17:109-19. 